\title{
Blockade of TRIM59 enhances esophageal cancer cell chemosensitivity to cisplatin by upregulating p53
}

\author{
RONGFENG LIU ${ }^{1}$, HONGCHEN LI ${ }^{2}$, YANZHAO XU ${ }^{3}, \mathrm{XING} \mathrm{LI}^{4}, \mathrm{XIAOJIN} \mathrm{GUO}{ }^{4}$, \\ JIAN SHI $^{1}$, YANZHI CUI ${ }^{1}$, ZHIYU WANG ${ }^{4}$ and JUNFENG LIU ${ }^{5}$ \\ ${ }^{1}$ Department of Oncology, Fourth Hospital of Hebei Medical University, Shijiazhuang, Hebei 050011; \\ ${ }^{2}$ Department of Pharmacology, Hebei Medical University, Shijiazhuang, Hebei 050017; \\ Departments of ${ }^{3}$ Thoracic Surgery, ${ }^{4}$ Immuno-Oncology and ${ }^{5}$ Third Department of Thoracic Surgery, \\ Fourth Hospital of Hebei Medical University, Shijiazhuang, Hebei 050011, P.R. China
}

Received February 16, 2020; Accepted September 17, 2020

DOI: $10.3892 / \mathrm{ol} .2020 .12267$

\begin{abstract}
Human esophageal cancer (hESC) cell motility adopts various modes, resulting in $\mathrm{hESC}$ progression and poor survival. However, how tripartite motif 59 (TRIM59), as the ubiquitination machinery, participates in hESC metastasis is not completely understood. The results indicated that TRIM59 was aberrantly upregulated in hESC tissues compared with adjacent healthy esophageal tissues, which was associated with poor survival and advanced TNM state among patients with hESC. Moreover, patients with hESC with higher TRIM59 expression displayed undetectable p53 expression, which contributed to enhanced progression and motility of hESC. At the molecular level, TRIM59 was indicated to be an E3 putative ubiquitin ligase that targeted the p53 protein, leading to increased degradation of $\mathrm{p} 53$, which resulted in decreased chemosensitivity to cisplatin. TRIM59 knockdown reduced TRIM59 expression, increased p53 protein expression, and decreased hESC cell viability, clone formation and migration compared with the small interfering RNA negative control (siNC) group. Furthermore, hESC cell lines were more sensitive to cisplatin in the TRIM59-knockdown group compared with the siNC group. The results indicated a relationship between TRIM59, p53 and the chemosensitivity of cisplatin. The present study suggested that TRIM59 may serve as a promising prognostic indicator for patients with hESC.
\end{abstract}

\section{Introduction}

Tripartite motif 59 (TRIM59) is a member of the TRIM family, which is characterized by an $\mathrm{N}$-terminal really interesting

Correspondence to: Professor Junfeng Liu, Third Department of Thoracic Surgery, Fourth Hospital of Hebei Medical University, 12 Jiankang Road, Shijiazhuang, Hebei 050011, P.R. China

E-mail: liujunfeng73@163.com

Key words: esophageal cancer, tripartite motif 59, cisplatin, p53, chemosensitivity new gene (RING)-finger domain, followed by two zinc-finger domains, B2 box and coiled-coil (1). TRIM59 protein has a domain at the C-terminal trans-membrane region (2). Ubiquitination serves a vital role in the degradation of several types of proteins that function in the intracellular signaling pathway, cell cycle, DNA repair and transcriptional regulation (2). E3 ubiquitin ligases directly recognize substrates on the basis of domain structures. There are two families of E3 ubiquitin ligases: The homologous to E6-AP $\mathrm{COOH}$ terminal family and the RING-finger-containing protein family (2). Due to the RING-finger domain, TRIM59 protein functions as a E3 ubiquitin ligase and can selectively target ubiquitin-modified proteins for proteasomes or degradation (2). In addition, TRIM proteins can positively or negatively promote carcinogenesis (3). The present study focused on the TRIM59 protein and its oncogenic activity in the promotion of human esophageal cancer (hESC) cell proliferation and metastasis. Matrix metalloproteinases (MMPs) play a key role in tumorigenesis, including early carcinogenesis events, tumor metastasis and invasion (4). Given the ability of tumor cells to infiltrate and disseminate, the role of MMP in cell invasion is important (5). Certain MMPs, such as MMP2 and MMP9 have special mechanism to help the invasion of cancer cells (5). MMP2 and MMP9 are family members of zinc-dependent endoproteases, responsible for degrading the extracellular matrix (ECM) by destroyingthe structure of various proteins (5). Degradation of the basement membrane and subsequently of the ECM is critical for invasion, and MMP2/9 are vital factors in providing the invasive and metastatic characteristics of malignant tumor cells by enabling their infiltration and migration in the process of EMT (5).

The $\mathrm{p} 53$ protein, the tumor suppressor gene tumor protein p53 (TP53), is mutated in 50\% of human cancer and serves a vital role in the response of malignant and non-transformed cells to several anticancer therapeutics, apoptosis, cell cycle arrest and DNA repair (6). A number of studies have demonstrated that mutant p53 expression promotes cancer cell proliferation, and wild-type p53 overexpression decreases transformed cancer cell proliferation, which is evidence of p53 serving as a tumor suppressor $(7,8)$. Several types of 553 mutations in cancer are point mutations in the DNA-binding 
domain; however, patients with Li-Fraumeni syndrome carry heterozygous germline p53 mutations and present with different types of cancer throughout their lifetime $(7,8)$. The mutant $\mathrm{p} 53$ proteins are not considered as functional proteins that can regulate the transcription of wild-type p53 target genes (6). Gene expression studies have reported that the wild-type p53 protein level was decreased in hESC compared with adjacent healthy tissues $(7,8)$. The reactivation of the $\mathrm{p} 53$ signaling pathway in response to several types of cancer may become an attractive means to understand hESC growth and mobility. However, why the wild-type p53 protein level is downregulated in hESC tissues and cell lines is not completely understood.

hESC is the most common type of digestive tumor, with high incidence $(572,000$ new cases in 2018) and mortality (509,000 mortalities in 2018) rates worldwide (9). Esophageal squamous cell carcinoma (ESCA) is the most common in the Taihang Mountain Area of China, with patients usually diagnosed at an advanced stage, due to the lack of no obvious symptoms at the early stage (10). Chemoradiation therapy is the most effective treatment strategy for hESC after surgery (10). Cisplatin is an effective chemotherapy drug, but cisplatin resistance frequently occurs in the cisplatin-based treatment of ESCA (11). Therefore, identifying the mechanism underlying cisplatin resistance in $\mathrm{hESC}$ requires further investigation.

\section{Materials and methods}

Surgical hESC samples. A total of 112 hESC and paired adjacent healthy tissues (5 $\mathrm{cm}$ away from tumor margin) were obtained by surgical removal from patients with hESC at the Forth Affiliated Hospital of Hebei Medical University (Shijiazhuang, China) between January 2015 and December 2017 (Table I). The clinicopathological characteristics of the patients are presented in Table I. A total of 85 men and 27 women were included in the present study. The median age was 60 years (age range, 50-80 years). All tissues were immediately frozen in liquid nitrogen and stored at $-80^{\circ} \mathrm{C}$. Written informed consent was provided by all patients or their relatives. There were several informed consents signed by patients' relatives because these patients had limited capacity, resulting in them being unable to sign the contents. The present study was approved by the Research Ethics Committees of the Forth Hospital of Hebei Medical University (Shijiazhuang, China).

Cell lines and antibodies. hESC cell lines (Eca109, KYSE150, KYSE30, KYSE510 and EC9706) and human esophageal epithelial cells (HEEC) were purchased from the American Type Culture Collection. hESC cells were maintained in DMEM (Gibco; Thermo Fisher Scientific, Inc.), while HEEC cells were maintained in RPMI-1640 (Corning Inc.) supplemented with $10 \%$ FBS (Gibco; Thermo Fisher Scientific, Inc.) and $1 \%$ penicillin-streptomycin (Gibco; Thermo Fisher Scientific, Inc.) at $37^{\circ} \mathrm{C}$ with $5 \% \mathrm{CO}_{2}$. The Eca109 cell line was a stable cisplatin-resistance cell line, as determined by continuous screening. The HEK293T cell line was purchased from the Cell Bank of the Institute for Biological Science, Chinese Academic of Science and cultured in DMEM (Gibco; Thermo Fisher Scientific, Inc.) supplemented with 10\% FBS and $1 \%$ penicillin-streptomycin (all purchased from Gibco; Thermo Fisher Scientific, Inc.) at $37^{\circ} \mathrm{C}$ with $5 \% \mathrm{CO}_{2}$.

The following antibodies were used for western blotting, immunofluorescence and immunoprecipitation. Primary antibodies targ-eted against: TRIM59 (cat. no. ab69639; 1:500 for western blotting, 1:300 for immunofluorescence and $10 \mu \mathrm{g} / \mathrm{ml}$ for immunoprecipitation; Abcam), p53 (cat. no. ab26; $10 \mu \mathrm{g} / \mathrm{ml}$ for western blotting and $5 \mu \mathrm{g} / \mathrm{ml}$ for immunoprecipitation; Abcam), matrix metallopeptidase (MMP) 9 (cat. no. ab38898; 1:1,000; Abcam), MMP2 (cat. no. ab97779; 1:1,000; Abcam), Bcl-2 (cat. no. ab32124; 1:1,000; Abcam), Tublin (cat. no. 801213; 1:5,000; BioLegend, Inc.) and GAPDH (cat. no. 649201; 1:5,000; BioLegend, Inc.). The secondary antibody (anti-Rabbit: Cat. no. 926-32211; anti-Mouse: Cat. no. 925-32210; anti-Human: Cat. no. 926-32232; 1:5,000; LI-COR) was purchased from LI-COR Biosciences. The FITC Annexin V Apoptosis Detection kit with PI (cat. no. 640914) was purchased from BioLegend, Inc.

Chemotherapy drug. Cisplatin was purchased from Nanjing Pharmaceutical Factory Co., Ltd.

Gene expression profiling of esophageal cancer. The Cancer Genome Atlas database (https://portal.gdc.cancer.gov) and Gene Expression Omnibus (GEO) database (dataset no. GEO30480, https://www.ncbi.nlm.nih.gov/geo) were used to analyze the gene expression profile of TRIM59 protein (12).

Immunoblotting, immunofluorescence, co-immunoprecipitation . For immunoblotting, cells were collected and total protein was isolated using high RIPA lysis buffer (cat. no. Ab156034; 1:10; Abcam) containing $1 \mathrm{mM}$ PMSF. Cell lysates were centrifuged at $12,000 \mathrm{x}$ g for $15 \mathrm{~min}$ at $4^{\circ} \mathrm{C}$, and total protein was quantified using the BCA method (cat. no. Ab102536; Abcam). Proteins $(40 \mu \mathrm{g})$ were added to $5 \mathrm{X}$ loading buffer for $5 \mathrm{~min}$ at $95^{\circ} \mathrm{C}$, separated via $12 \%$ SDS-PAGE and transferred to PVDF membranes (Immobilon-FL; EMD Millipore). The membranes were blocked with 5\% skimmed milk powder in PBS for $2 \mathrm{~h}$ at room temperature. The membranes were incubated with primary antibodies overnight at $4^{\circ} \mathrm{C}$. The second antibodies were used to incubate the membrane for $2 \mathrm{~h}$ at $25^{\circ} \mathrm{C}$. TRIM59, p53, MMP9 and MMP2 protein bands were visualized using the Odyssey Imaging Systems (ODYSSEY ${ }^{\circledR}$, LI-COR Biosciences) and Application software (version 2.1.12; LI-COR Biosciences).

For immunofluorescence, following fixation with $4 \%$ paraformaldehyde for $2 \mathrm{~h}$ at $4^{\circ} \mathrm{C}$, frozen sections embedded with tissue freezing medium (cat. no. 03812525; Leica Biosystem Richmond, Inc.) were permeabilized with $0.3 \%$ Triton X-100/PBS for $1 \mathrm{~h}$ at $37^{\circ} \mathrm{C}$ and blocked with $10 \%$ normal goat serum (cat. no. Ab7481; 1:1,000; Abcam) in PBS for $30 \mathrm{~min}$ at $37^{\circ} \mathrm{C}$. Tissue slides were incubated with primary antibodies overnight at $4^{\circ} \mathrm{C}$. Subsequently, tissues were incubated with a fluorescent secondary antibody (cat. no. 111-165-003; 1:400; Jackson ImmunoResearch Inc.) for $1 \mathrm{~h}$ at room temperature, followed by washing three times with PBS (10 min per time). Nuclear staining was performed using DAPI for $10 \mathrm{~min}$ in the dark at room temperature. Following washing one time with PBS and drying, SlowFade ${ }^{\mathrm{TM}}$ Diamond Antifade Mountant solution (cat. no. S36963; Thermo Fisher 
Table I. Characteristics of patients with human esophageal cancer.

\begin{tabular}{|c|c|c|c|c|c|}
\hline Characteristics & $\mathrm{n}$ & $\begin{array}{l}\text { TRIM59 negative/low } \\
\text { expression }(n=42)\end{array}$ & $\begin{array}{l}\text { TRIM59 high } \\
\text { expression }(n=70)\end{array}$ & P-value & $\begin{array}{l}\text { Statistical } \\
\text { method }\end{array}$ \\
\hline Age (years) & & & & 0.658 & \\
\hline$<60$ & 84 & 29 & 55 & & Mann-Whitney U test \\
\hline$\geq 60$ & 28 & 13 & 15 & & \\
\hline Gender & & & & 0.284 & \\
\hline Male & 85 & 26 & 59 & & Mann-Whitney U test \\
\hline Female & 27 & 16 & 11 & & \\
\hline Histological type & & & & 0.987 & \\
\hline Squamous cell carcinoma & 110 & 40 & 70 & & Mann-Whitney U test \\
\hline Adenocarcinomas & 2 & 2 & 0 & & \\
\hline Localization & & & & 0.692 & \\
\hline Upper & 20 & 8 & 12 & & Kruskal-Wallis test \\
\hline Middle & 56 & 14 & 42 & & \\
\hline Lower & 36 & 20 & 16 & & \\
\hline Invasive depth & & & & $0.000^{\mathrm{a}}$ & \\
\hline $\mathrm{T} 1-2$ & 20 & 11 & 9 & & Kruskal-Wallis test \\
\hline T3 & 50 & 25 & 25 & & \\
\hline $\mathrm{T} 4$ & 42 & 6 & 36 & & \\
\hline pTNM stage & & & & $0.000^{\mathrm{a}}$ & \\
\hline $\mathrm{I}$ & 20 & 8 & 12 & & Kruskal-Wallis test \\
\hline II & 30 & 18 & 12 & & \\
\hline III & 56 & 16 & 40 & & \\
\hline IV & 6 & 0 & 6 & & \\
\hline Lymph node metastasis & & & & $0.003^{\mathrm{a}}$ & \\
\hline Absence & 55 & 14 & 41 & & Mann-Whitney U test \\
\hline Presence & 57 & 28 & 29 & & \\
\hline
\end{tabular}

${ }^{\mathrm{a}} \mathrm{P}<0.05$. Statistical analyses were performed using the $\chi^{2}$ test.

Scientific, Inc.) was added to the tissues and cover glasses were placed on the slides. Stained slides were observed under a fluorescence microscope (magnification, x20) and analyzed using LAS AF software (version 2.5.2 build 6939; Leica Microsystems).

For immunoprecipitation assays, at $36 \mathrm{~h}$ post-transfection (Lipofectamine ${ }^{\mathrm{TM}} 2000$ Transfection Reagent; cat. no. 11668019; Thermo Fisher Scientific, Inc.), cells were rinsed with PBS and lysed in RIPA buffer (cat. no. Ab156034; 1:10; Abcam) supplemented with a protease and phosphatase inhibitor cocktail (cat. no. 78427; 1:100; Thermo Fisher Scientific, Inc.) for $30 \mathrm{~min}$ on ice. Whole cell extract was incubated with indicated primary antibodies or with anti-IgG (cat. no. Ab181236; 1:10,000; Abcam) in a vertical rocker overnight at $4^{\circ} \mathrm{C}$. Protein A/G beads (50 $\mu$; cat. no. 20421; Thermo Fisher Scientific, Inc.) were added and incubated for another $4 \mathrm{~h}$ at $4^{\circ} \mathrm{C}$ to pull down the TRIM59 or p53 protein. Following incubation, the agarose gels were washed five times with lysis buffer and boiled for $5 \mathrm{~min}$ at $98^{\circ} \mathrm{C}$ with $4 \mathrm{X}$ SDS loading buffer, and then subjected to $12 \%$ SDS-PAGE. Proteins were transferred onto PVDF membranes (Immobilon-FL; EMD Millipore). Membranes were blocked with 5\% BSA (cat. no. V900933; Sigma-Aldrich; Merck KGaA) at $25^{\circ} \mathrm{C}$ for $2 \mathrm{~h}$ and incubated with primary antibodies overnight at $4^{\circ} \mathrm{C}$. Following the primary incubation, membranes were incubated with anti-rabbit second antibody (cat. no. 926-32211; 1:5,000) or anti-mouse second antibody (cat. no. 925-32210; $1: 5,000)$ at $25^{\circ} \mathrm{C}$ for $1.5 \mathrm{~h}$. The membranes were detected using an Odyssey infrared scanner (LiI-COR Biosciences) and assayed using Application software (version 2.1.12; LI-COR Biosciences).

Wound healing and clone formation assays. Eca109 cells were plated in 6-well plates. At 90-100\% confluence, cells were cultured in medium containing 0.5\% FBS (cat. no. 10099141; Gibco; Thermo Fisher Scientific, Inc.) for 24 h. Subsequently, cells were treated with $20 \mu \mathrm{g} / \mathrm{ml}$ mitomycin C (cat. no. 4287; Merck KGaA) for $4 \mathrm{~h}$ at $37^{\circ} \mathrm{C}$. Subsequently, a wound was scratched using sterile pipette tips in vertical and horizontal directions. Cells were gently washed three times with PBS and cultured with complete medium containing $10 \%$ FBS (Gibco; Thermo Fisher Scientific, Inc.) (12). Notably, cells in $10 \%$ FBS-supplemented medium exhibited a significantly enhanced proliferative ability after the aforementioned 
treatments. Thus, the present study investigated whether $10 \%$ FBS-supplemented medium can improve cell viability while maintaining effective propagation. The width of the scratched area was recorded and photographed using an inverted light-field microscope (magnification, x10) every $12 \mathrm{~h}$. The quantitative analysis of the scratch was computed and the differentiated images were presented when cells were cultured for $48 \mathrm{~h}$ following transfection.

For clone formation assay, cells were seeded $\left(0.5-1 \times 10^{4}\right.$ cells/well) into 6 -well plates and cultured for $\sim 2$ weeks. When clones grew to $>50 \mu \mathrm{m}$ in diameter, clones were defined and subsequently fixed with $4 \%$ paraformaldehyde for $30 \mathrm{~min}$ at $25^{\circ} \mathrm{C}$ and stained with $0.5 \%$ crystal violet staining solution for $30 \mathrm{~min}$ at $25^{\circ} \mathrm{C}$. Cells were observed under an inverted light-field microscope (magnification, x10).

Cell Counting Kit-8(CCK-8). A CCK-8 assay (cat.no.HY-K0301; MedChemExpress) was performed to evaluate cell proliferation according to the manufacturer's instructions. Cells were seeded $\left(1 \times 10^{5}\right)$ into 96-well plates and incubated for $0,24,48$ and $72 \mathrm{~h}$ at $37^{\circ} \mathrm{C}$. Subsequently, $10 \mu \mathrm{l} \mathrm{CCK}-8$ reagent was added into each well and incubated at $4 \mathrm{~h}$ at $37^{\circ} \mathrm{C}$. Absorbance was measured at a wavelength of $450 \mathrm{~nm}$ using a FLUOstar Omega microplate reader (BMG Labtech $\mathrm{GmbH})$.

RNA extraction and reverse transcription-quantitative $P C R$ (RT-qPCR). Total RNA was isolated from tissues and cells using the RNeasy mini kit (Qiagen AB) according to the manufacturer's instructions. The remaining DNA was removed by adding DNase I. Total RNA was reverse transcribed into cDNA using the Advantage ${ }^{\circledR}$ RT-for-PCR kit (cat. no. 639505; Clontech Laboratories, Inc.). The reaction conditions for reverse transcription were $37^{\circ} \mathrm{C}$ for $30 \mathrm{~min}$ and $85^{\circ} \mathrm{C}$ for $10 \mathrm{sec}$. Subsequently, qPCR was performed using the Real-Time PCR Detection system (Bio-Rad Laboratories, Inc.) with TB Green ${ }^{\circledR}$ Premix Ex Taq ${ }^{\mathrm{TM}}$ (cat. no. RR420Q; Takara Bio Inc.). The following thermocycling conditions were used for qPCR: $95^{\circ} \mathrm{C}$ for $50 \mathrm{sec} ; 40$ cycles at $95^{\circ} \mathrm{C}$ for $5 \mathrm{sec}$ and $60^{\circ} \mathrm{C}$ for $50 \mathrm{sec}$. The following primers were used for qPCR: TRIM59 forward, 5'-CCTGTGTTTGAGATA GATTTAAGAGC-3' and reverse, 5'-GCAACAAGGTGA GACCCAGT-3'; GAPDH forward, 5'-TTGATGGCAACA ATCTCCAC-3' and reverse, 5'-CGTCCCGTAGACAAA ATGGT-3'; Bcl-2 forward, 5'-ACTGAGTACCTGAACCGG CA-3' and reverse, 5'-GAAATCAAACAGAGGCCGCAT-3'; MMP2 forward, 5'-ACAAAGGGATTGCCAGGACC-3' and reverse, 5'-CGGTGGCCTGGGGTTTG-3'; MMP9 forward, 5'-CCTGGGCAGATTCCAAACCT-3' and reverse, 5'-CAA AGGCGTCGTCAATCACC-3'; p53 forward, 5'-AAGTCT AGAGCCACCGTCCA-3' and reverse, 5'-CAATCCAGG GAAGCGTGTCA-3'. mRNA expression levels were quantified using the $2^{-\Delta \Delta \mathrm{Cq}}$ method (12) and normalized to the internal reference gene GAPDH.

RNA interference. TRIM59 siRNA, and scrambled NC were synthesized by Sangon Biotech Co., Ltd. Eca109 cells (1x10 $/$ well) were seeded and cultured for $24 \mathrm{~h}$. Subsequently, Eca109 cells were transfected with $2 \mu \mathrm{g}$ TRIM59 siRNA or NC using Lipofectamine ${ }^{\circledR} 3000$ (cat. no. L3000015; Invitrogen; Thermo Fisher Scientific, Inc.) according to the manufacturer's instructions. At $48 \mathrm{~h}$ post-transfection, transfection efficiency was assessed via RT-qPCR, fluorescence microscopy and western blotting. The method of siTP53transfection performed according to the same protocol described for TRIM59 siRNA transfection. The sequences of the siRNAs were as follows: siTRIM59a, 5-CCCUGA ACAUUACAG GCA ATT-3'; siTRIM59b, CCAGCATGTACAGATCTTGAAA; NC forward, 5'-UUCUCCGAACGUGUCACGUTT-3' and reverse, 5'-ACG UGACACGUUCGGAGAATT-3'; and siTP53, 5'-CUACUU CCUGAAAACAACG-3'; and NC for TP53, 5'-UUCUCCGAA CGUGUCACGUTT-3'.

Statistical analysis. All experiments were performed in triplicate and data are presented as the mean \pm standard deviation. Statistical analyses were performed using GraphPad Prism (version 6; GraphPad Software, Inc.) and SPSS (version 21.0; IBM Corp.). A $\chi^{2}$ test was used to examine the association between TRIM59 expression and clinicopathological features. The Kaplan-Meier method with log-rank tests was used to estimate overall patient survival. Comparisons between two groups were analyzed using the paired Student's t-test. Comparisons among multiple groups were analyzed using one-way analysis of variance, followed by Tukey's post hoc test. $\mathrm{P}<0.05$ was considered to indicate a statistically significant difference.

\section{Results}

Upregulation of TRIM59 in hESC tissues and cell lines, and its association with poor prognosis. In a previous study, TRIM59 promoted the growth of several types of cancer, including non-small cell lung cancer (12); however, its function in hESC cells is not completely understood. To investigate the significance of TRIM59 in hESC cells, the GSE30480 dataset was downloaded from the GEO database and used to analyze the multiple microarray dataset from Tan et al (12). The results indicated that TRIM59 mRNA levels were enhanced in hESC cells derived from tumor tissues compared with hESC cells derived from healthy adjacent tissues (Fig. 1A). Moreover, by analyzing the relevant clinical data, the results indicated that increased TRIM59 expression was associated with decreased survival in patients with hESC (Fig. 1A). Collectively, the results suggested that the high expression of TRIM59 in patients with hESC was positively associated with poor prognosis.

To verify the results of the microarray analysis, the mRNA and protein expression levels of TRIM59 were detected in hESC tissues and matched adjacent healthy tissues via RT-qPCR. The RT-qPCR results indicated that TRIM59 was significantly increased in hESC tissues compared with the adjacent healthy esophageal tissues (Fig. 1D). The mRNA expression levels of TRIM59 were detected in a hESC cell line (Eca109) using HEEC as a non-malignant control. The results indicated that TRIM59 expression was significantly increased in Eca109 cells compared with HEEC cells (Fig. 1B).

The protein expression levels of TRIM59 in hESC tissues were further verified via western blotting. Among the 112 patients, TRIM59 protein expression was detected in 112 biopsy samples compared with the matched adjacent 
A

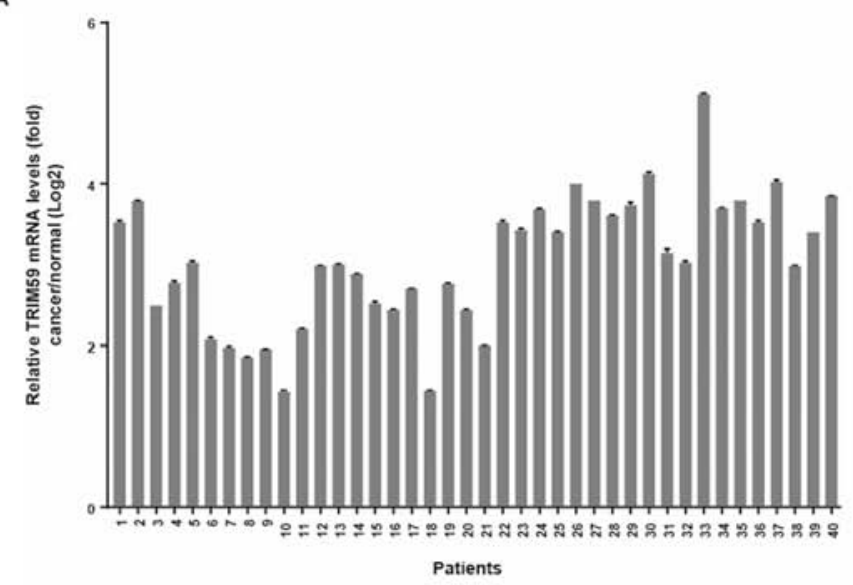

C

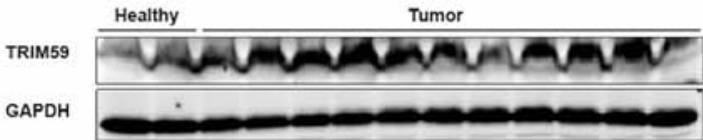

B

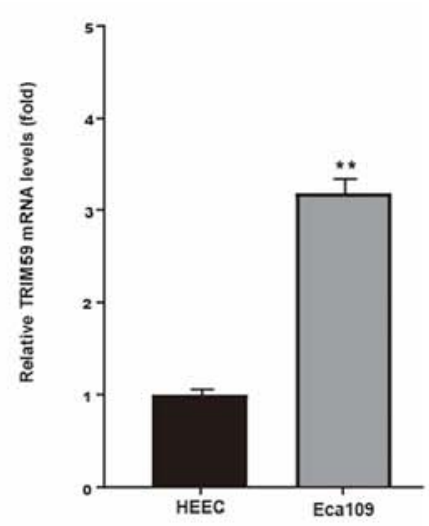

D

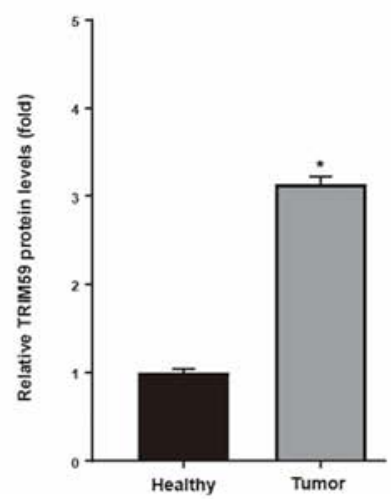

E
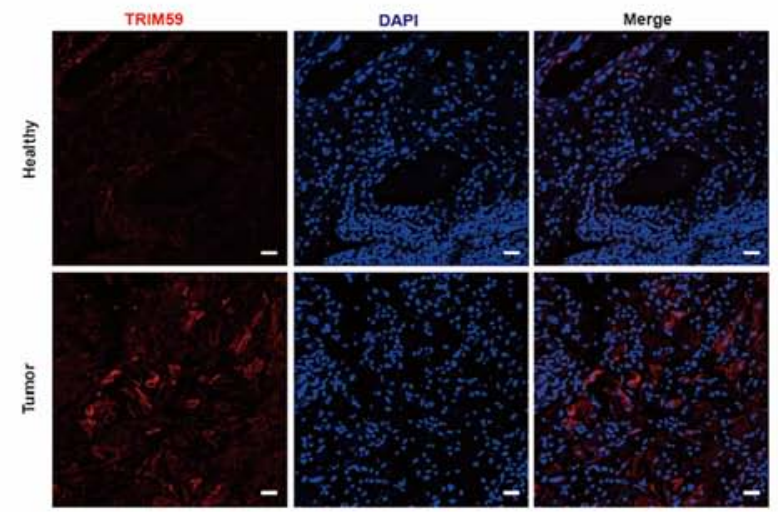
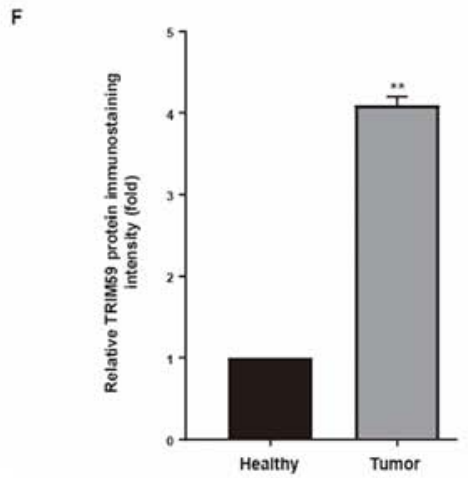

G

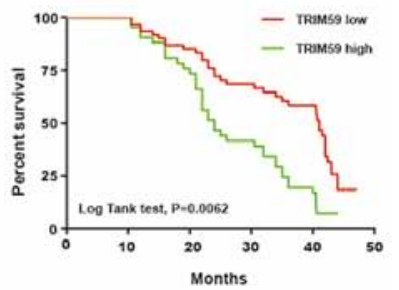

Figure 1. Higher TRIM59 mRNA expression in tumor tissues and cell lines. (A) TRIM59 mRNA levels in human tumor tissues and matched healthy tissues from 40 patients were determined via reverse transcription-quantitative PCR. The positive log2 value indicated higher expression levels of TRIM59 in human tumor tissues compared with matched healthy tissues. (B) TRIM59 mRNA levels in a hESC cell line and matched human esophageal epithelial cells. TRIM59 protein expression levels in human tumor tissues and matched healthy tissues were (C) determined via western blotting and (D) semi-quantified $(\mathrm{n}=112)$. TRIM59 protein expression levels in human tumor tissues and matched healthy tissues were (E) determined via immunofluorescence staining (scale bar, $50 \mu \mathrm{m}$ ) and (F) quantified. (G) Kaplan-Meier survival curve for patients with hESC. Higher TRIM59 expression levels were associated with poor patient survival. " $\mathrm{P}<0.05$ and $^{* *} \mathrm{P}<0.01$ vs. HEEC or healthy. TRIM59, tripartite motif 59; hESC, human esophageal cancer; HEEC, human esophageal epithelial cells. 
healthy tissues, and the representational figure using the tissues from 11 cancer tissues and 2 adjacent normal tissues (Fig. 1C and D). Therefore, the high protein expression level of TRIM59 in hESC may originate from the upregulation of TRIM59 transcription.

To further investigate the association between TRIM59 and hESC, immunofluorescence staining of TRIM59 was performed on primary tumors and matched adjacent healthy tissues. Among the 112 specimens, 112 biopsy samples contained tumor and matched adjacent tissues. TRIM59 expression was significantly increased in hESC tissues compared with adjacent healthy tissues, as determined by immunofluorescence staining (Fig. 1E and F). In addition, Kaplan-Meier analysis was conducted to assess the association between high TRIM59 expression and patient survival. High TRIM59 expression was associated with decreased patient survival (Fig. 1G).

TRIM59 promotes hESC cell migration and invasion in vitro. To determine the clinical characteristics and prognostic value of TRIM59 expression in hESC, its potential biological function was further explored. First, the protein expression levels of TRIM59 were detected in Eca109, EC9706, HYSE30 and HYSE150 cell lines. The results indicated that TRIM59 expression levels were significantly higher in Eca109 cells compared with the HEEC cell line (Fig. 2A and B), which was observed in all four cell lines. Higher TRIM59 expression levels were observed in Eca109 cells, whereas lower TRIM59 expression levels were observed in EC9706, HYSE30 and HYSE150 cells. Increased TRIM59 protein expression levels in Eca109 cells were consistent with the trends of increased TRIM59 expression in tumor specimens compared with healthy tissues identified via western blotting and RT-qPCR.

To explore the effect of TRIM59 knockdown on cell proliferation, two TRIM59-targeting siRNAs (siRNAa and siRNAb) or scramble siRNA were used to examine the biological function of TRIM59 in hESC cell lines. The results suggested that both TRIM59-targeting siRNAs significantly decreased TRIM59 protein expression levels in the Eca109 cell line compared with the scramble siRNA. Therefore, both siRNAs were used in subsequent experiments due to their knockdown effect. The RT-qPCR and western blotting results suggested that TRIM59 siRNAs significantly decreased the mRNA and protein expression levels of TRIM59 in the Eca109 cell line compared with siNC (Fig. 2C-E).

Following TRIM59 knockdown, alterations in cell numbers, migration and viability were investigated using a hESC cell line. To assess cell proliferation, colony formation assays were performed. The results indicatedthat TRIM59 knockdown significantly decreased the colony formation ability of the Eca109 cell line compared with the siNC group (Fig. 2F and G).

The clinical investigation and analysis of hESC indicated that patients with higher TRIM59 expression displayed poor survival (Fig. 1G). First, to examine the biological function of TRIM59 in tumor migration, wound healing assays were performed. The results suggested that TRIM59 knockdown significantly inhibited Eca109 cell migration by $50-60 \%$ compared with the siNC group (Fig. 2H and I). Subsequently, the effects of TRIM59 knockdown on Eca109 cell viability were assessed by performing a CCK-8 assay. The results indicated that TRIM59-knockdown Eca109 cells displayed significantly reduced cell viability compared with the siNC group at 24, 36 and $48 \mathrm{~h}$ (Fig. 2J).

Based on the association between cell proliferation and migration-related proteins, the association between matrix metalloproteinases, including MMP2 and MMP9, and TRIM59 was examined in Eca109 cells. RT-qPCR and western blotting were performed to quantify MMP2 and MMP9 expression levels in TRIM59-knockdown Eca109 cells.

MMP2 and MMP9 mRNA expression levels were significantly decreased in TRIM59-knockdown cells compared with the siNC group (Fig. 2K). The results also indicated that TRIM59 knockdown significantly reduced MMP2 and MMP9 protein expression levels compared with the siNC group (Fig. 2L and M). The results suggested that TRIM59 promoted hESC cell proliferation, migration and invasion.

TRIM59 knockdown enhances Ecal09 chemosensitivity to cisplatin. hESC progression and chemoresistance serve important roles in patient survival and prognosis (11). It was predicted that cisplatin, the most prevalent first-line treatment for hESC, would become non-sensitive to tumor cells, resulting in treatment failure. Therefore, elucidating the mechanism underlying cisplatin in hESC is important. The results indicated that TRIM59 expression was significantly increased in hESC tissues compared with healthy esophageal epithelial tissues (Fig. 1C and D). Compared with the siNC group, TRIM59 knockdown inhibited esophageal cancer proliferation and migration, which indicated that the chemosensitivity effects of TRIM59 in hESC should be investigated further. Therefore, it was hypothesized that enhancing TRIM59 promoted hESC progression and contributed to chemoresistance to cisplatin. Eca109 cells were selected as the cisplatin-resistant hESC cell line. Subsequently, drug sensitivity was measured to assess TRIM59 knockdown-mediated alterations in cisplatin resistance. Of note, cisplatin-induced cell viability in the siTRIM59-knockdown group was significantly lower compared with the siNC + cisplatin group. Theresults also suggested that cisplatin enhanced hESC cell death in siTRIM59-transfected cells (Fig. 3A). To examine whether cisplatin treatment induced Eca109 cell apoptosis, cells were transfected with siTRIM59 or siNC. Following incubation for $48 \mathrm{~h}$ at $37^{\circ} \mathrm{C}$, cells were treated with cisplatin for $48 \mathrm{~h}$. Cell apoptosis was analyzed via immunostaining with Annexin V and PI. The number of apoptotic cells was significantly increased in the siTRIM59 + cisplatin group compared with the siTRIM59 + DMSO and si-NC groups (Fig. 3B-D). Collectively, the results indicated that Eca109 cells pretreated with siTRIM59 displayed increased cisplatin-related cell apoptosis.

TRIM59 knockdown regulates the expression levels of antiapoptotic and migration-related proteins in response to cisplatin. To investigate whether the combined treatment of siTRIM59 and cisplatin inhibited Eca109 cell migration, the expression levels of antiapoptotic and migration-related proteins were compared among the combined, cisplatin, siTRM59 and siNC groups. The protein expression level of Bcl-2 was determined via western blotting to explore the molecular mechanism underlying apoptotic upregulation 


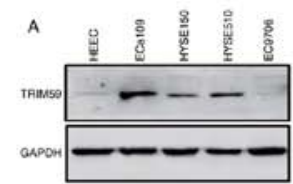

c
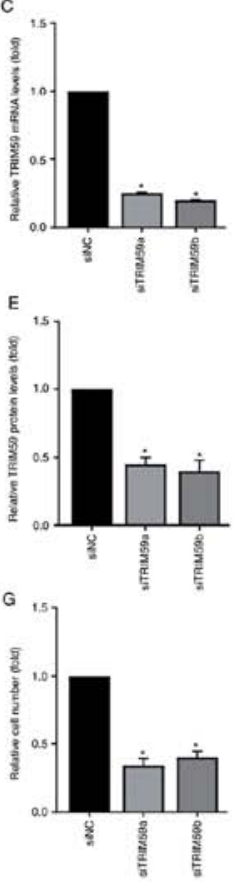

$\mathrm{H}$
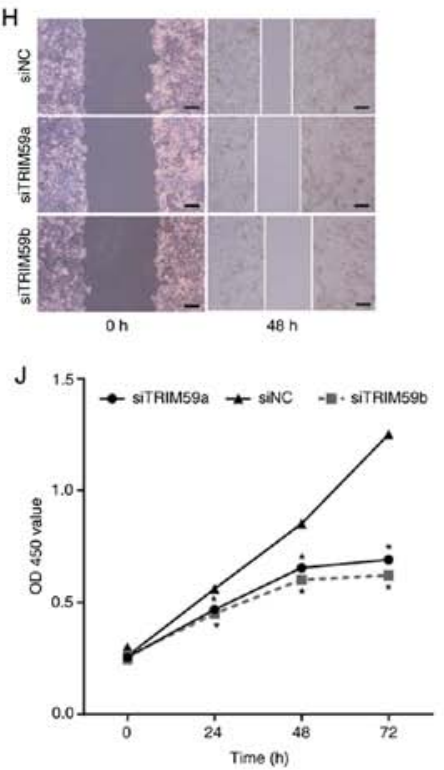

L

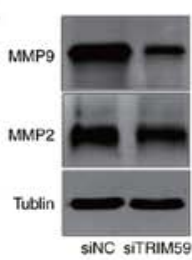

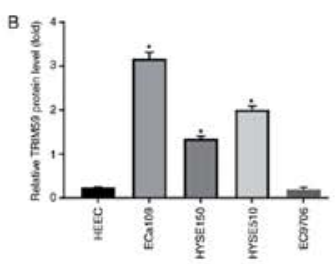

D
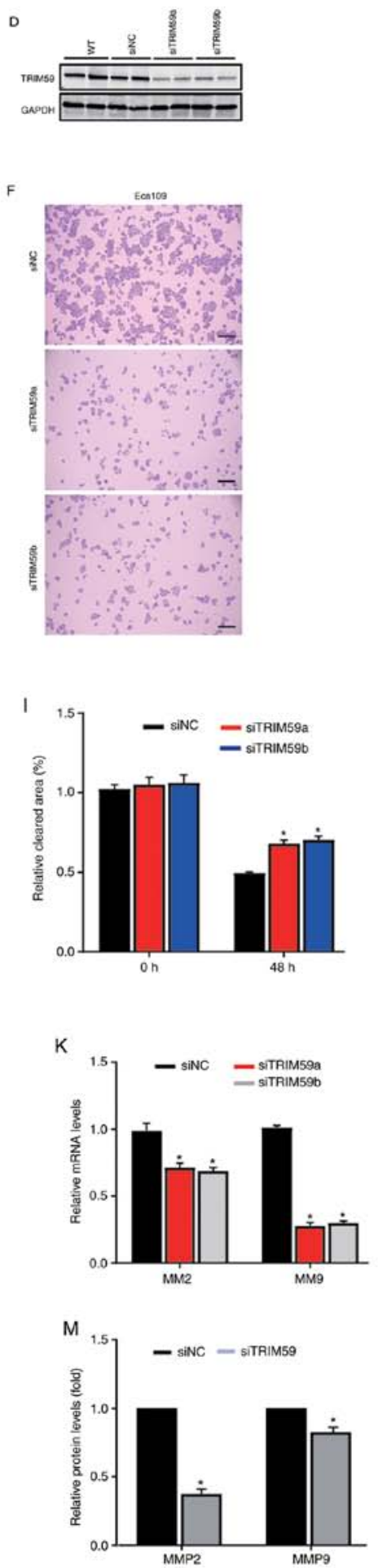

Figure 2. TRIM59 knockdown inhibits hESC cell migration and invasion. TRIM59 protein expression levels were (A) determined via western blotting and (B) semi-quantified. (C) At 48 h post-transfection, TRIM59 mRNA expression levels were measured via reverse transcription-quantitative PCR. At 48 h post-transfection, TRIM59 protein expression levels were (D) determined via western blotting and (E) semi-quantified. The effect of TRIM59 knockdown on hESC cell proliferation was (F) determined by performing a colony formation assay (scale bar, $200 \mu \mathrm{m}$ ) and (G) quantified. The effect of TRIM59 knockdown on hESC cell migration was (H) assessed by performing a wound healing assay (scale bar, $200 \mu \mathrm{m}$ ) and (I) quantified. (J) Eca109 cell viability was measured at different time points using a Cell Counting Kit-8 assay. (K) The effect of TRIM59 knockdown on MMP2 and MMP9 mRNA expression levels. The effect of TRIM59 knockdown on MMP2 and MMP9 protein expression levels was (L) assessed via western blotting and (M) semi-quantified. ${ }^{*} \mathrm{P}<0.05$ vs. Eca109 or siNC. TRIM59, tripartite motif-containing 59 ; hESC, human esophageal cancer; MMP, matrix metallopeptidase; si, small interfering RNA; NC, negative control; WT (function as an ineffective proof of siNC), wild-type; OD, optical density. 

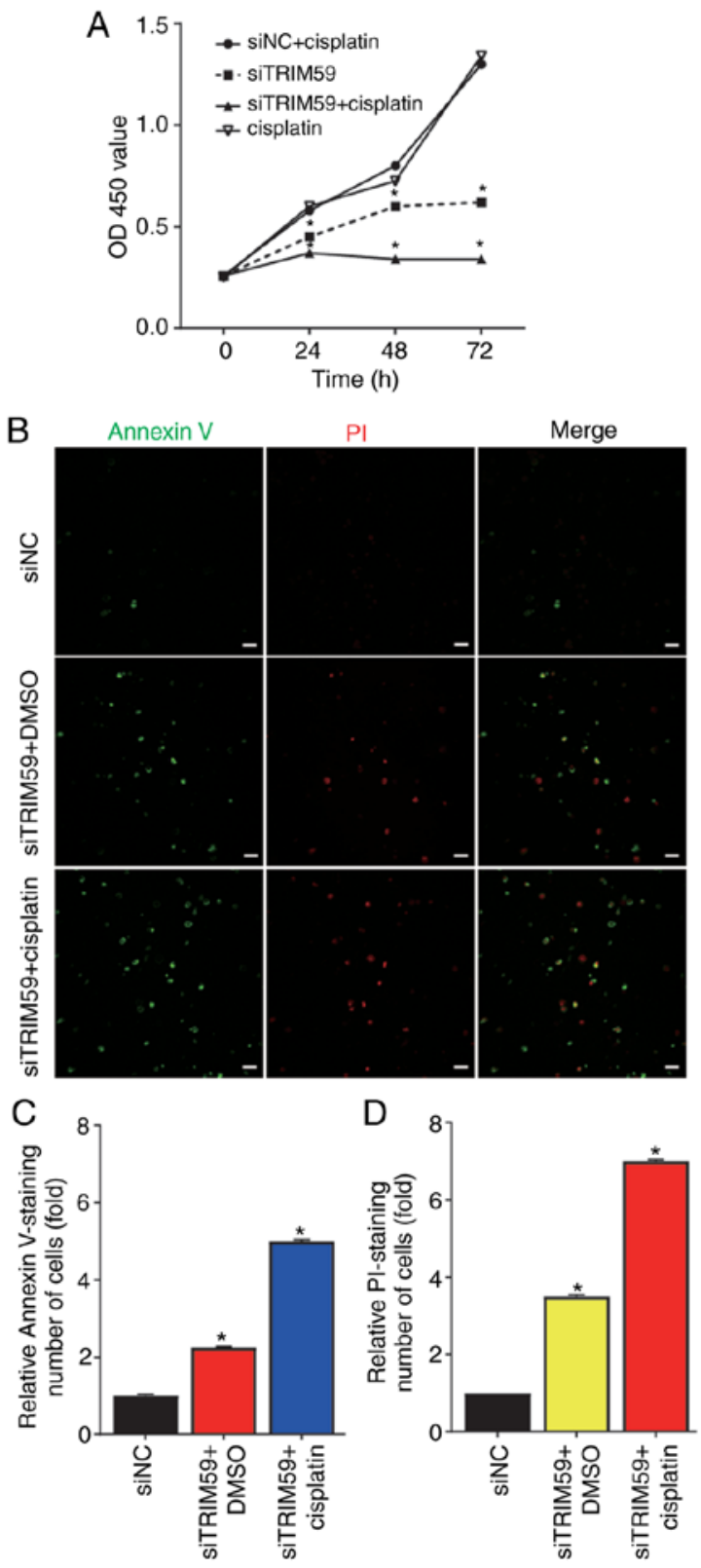

Figure 3. TRIM59 knockdown enhances Eca109 cell chemo-sensitivity to cisplatin. (A) Cell viability was measured at different time points using a Cell Counting Kit- 8 assay. ${ }^{*} \mathrm{P}<0.05$ vs. siNC + cisplatin. (B) Cell apoptosis was detected via Annexin V and PI staining (scale bar, $50 \mu \mathrm{m}$ ). Relative (C) Annexin V and (D) PI staining was quantified. ${ }^{*} \mathrm{P}<0.05$ vs. siNC. TRIM59, tripartite motif 59; OD, optical density; si, small interfering RNA; $\mathrm{NC}$, negative control.

in Eca109 cells. The results indicated that the antiapoptotic protein Bcl-2 was significantly upregulated in the siTRIM59 group compared with the siNC group. Furthermore, a slightly higher protein expression level of Bcl-2 was observed in the siTRIM59 + cisplatin group compared with the siNC group (Fig. 4A-C). To better understand the observed synergy between TRIM59 knockdown and cisplatin, expressive difference of Bcl-2 between siTRIM59 + cisplatin group and siTRIM59 was assessed, and the results demonstrated that the union group exhibited higher Bcl-2 levels. The expression levels of the migration-related proteins MMP2 and MMP9 were also measured via RT-qPCR and western blotting in the siTRIM59, siNC and combined treatment groups. The results suggested that MMP2 and MMP9 expression levels were significantly decreased in the siTRIM59 group compared with the siNC group (Fig. 4D-F). Moreover, the protein expression levels of MMP2 and MMP9 were further downregulated in the siTRIM59 + cisplatin group compared with the siNC group. To determine the synergy function of decreased invasion in union group of siTRIM59 and cisplatin, the expressive difference of MMP2 and MMP9 between the union and siTRIM59 groups was compared, and the results demonstrated that the union group exhibited lower MMP2 and MMP9 levels. Collectively, the results indicated that TRIM59 knockdown increased the protein expression levels of the antiapoptotic protein Bcl-2 and restrained tumor cell migration by decreasing MMP2 and MMP9 expression levels.

TRIM59 expression is negatively associated with p53 in hESC samples and Ecal09 cells. To understand the molecular mechanism underlying TRIM59-mediated promotion of hESC, several proteins previously reported to serve a critical role in esophageal tumorigenesis and possibly be regulated by TRIM59 were examined (12). Since several studies have identified that p53 is lowly expressed in $\operatorname{hESC}(13,14)$, it was hypothesized that $\mathrm{p} 53$ expression was correlated with TRIM59 expression. Therefore, p53 expression was detected in hESC tissues and Eca109 cells to explore the mechanism underlying TRIM59-mediated promotion of hESC proliferation. The RT-qPCR results indicated that p53 mRNA expression levels were significantly decreased in patients with higher TRIM59 expression levels compared with the control group (Fig. 5A). In addition, the protein expression level of p53 was analyzed by performing western blotting on surgically dissected hESC tissues. Consistently, p53 protein expression levels in hESC tissues were significantly lower compared with adjacent healthy tissues (Fig. 5B and C). Based on measurements in tissues isolated from patients, a strong negative association was identified between TRIM59 and p53 protein levels (Figs. 1C and 5B). To determine whether TRIM59 knockdown affected p53 expression, RT-qPCR was performed to detect the mRNA expression levels of TP53 in the different treatment groups, including siTRIM59 and siTP53 double-knockdown cells. The transfection efficiency of siTP53 was confirmed via RT-qPCR and western blotting (Fig. S1). The results from the Eca109 cell line suggested that TRIM59 knockdown significantly increased TP53 mRNA expression levels compared with the siNC group, which was inhibited by co-transfection with siTP53 (Fig. 5D).

The western blotting results further suggested the negative role of TRIM59 in the regulation of p53; therefore, the impact of TRIM59 expression on p53 was investigated in Ec109 cells (Fig. 5E and F). In combination, the results suggested that TRIM59 was highly expressed in patients with hESC, resulting in accelerated tumor growth and migration by inhibiting the p53 tumor-suppressor signaling pathway.

Subsequently, the molecular mechanism underlying TRIM59-mediated promotion of p53 degradation required further investigation. Therefore, whether TRIM59 interacted with p53 was investigated. A co-immunoprecipitation assay was designed to detect the physical association between TRIM59 and p53 using $293 \mathrm{~T}$ cells transfected with TRIM59 and p53 plasmids. The results indicated that TRIM59 bound to p53 

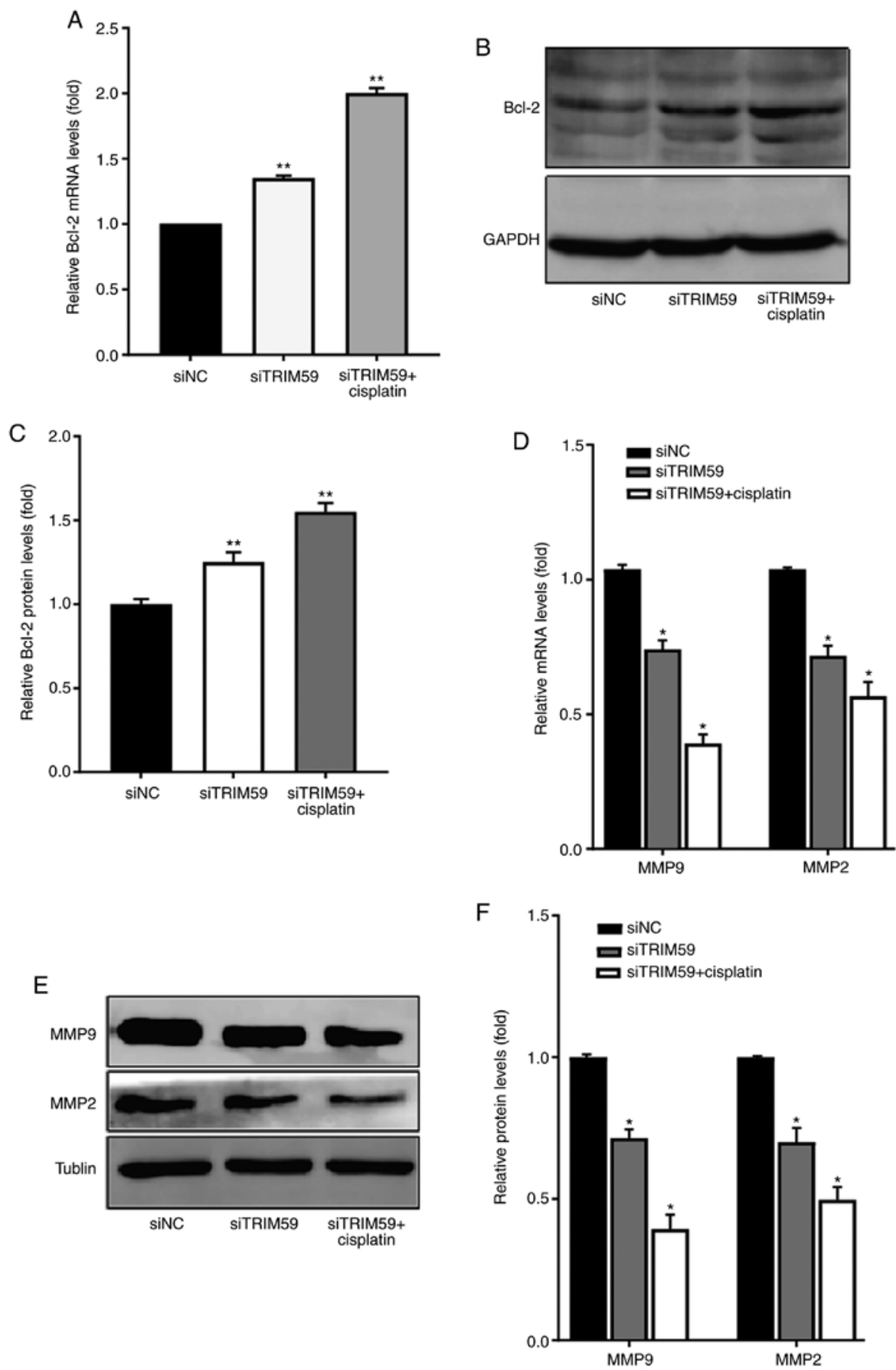

Figure 4. TRIM59 knockdown regulates the expression levels of apoptosis- and migration-related proteins in response to cisplatin. (A) Bcl-2 mRNA expression levels $(n=3)$. Bcl-2 protein expression levels were (B) determined via western blotting and (C) semi-quantified. (D) The mRNA expression levels of MMP2 and MMP9. MMP2 and MMP9 protein expression levels were (E) determined via western blotting and (F) semi-quantified. ${ }^{*} \mathrm{P}<0.05$ and ${ }^{* *} \mathrm{P}<0.01$ vs. siNC. TRIM59, tripartite motif 59; MMP, matrix metallopeptidase; si, small interfering RNA; NC, negative control.

(Fig. 5G). Collectively, the results indicated that TRIM59 was a negative regulator of $\mathrm{p} 53$, which enhanced $\mathrm{p} 53$ degradation.

\section{Discussion}

hESC is the leading cause of cancer-related mortality worldwide, particularly in men, and is the fifth most common type of cancer, with $>400,000$ cases of mortality in the first 5 years from diagnosis worldwide $(15,16)$. In-depth studies are required to explore and clarify the pathogenesis of hESC, in order to provide the tools for a more accurate diagnosis and effective treatment strategy. TRIM59, an important member of the TRIM protein family, is upregulated in hESC tissues and serves as an oncogene by promoting proliferation and migration (12). In addition, TRIM59 overexpression significantly increased cisplatin resistance in hESC cells. Therefore, TRIM59 was identified as a prognostic or predictive biomarker of hESC (12). However, identifying the mechanism underlying TRIM59 to develop advanced treatment strategies for hESC is important.

TRIM59, a member of the TRIM family of proteins, serves a crucial role in the proliferation and metabolism of several 

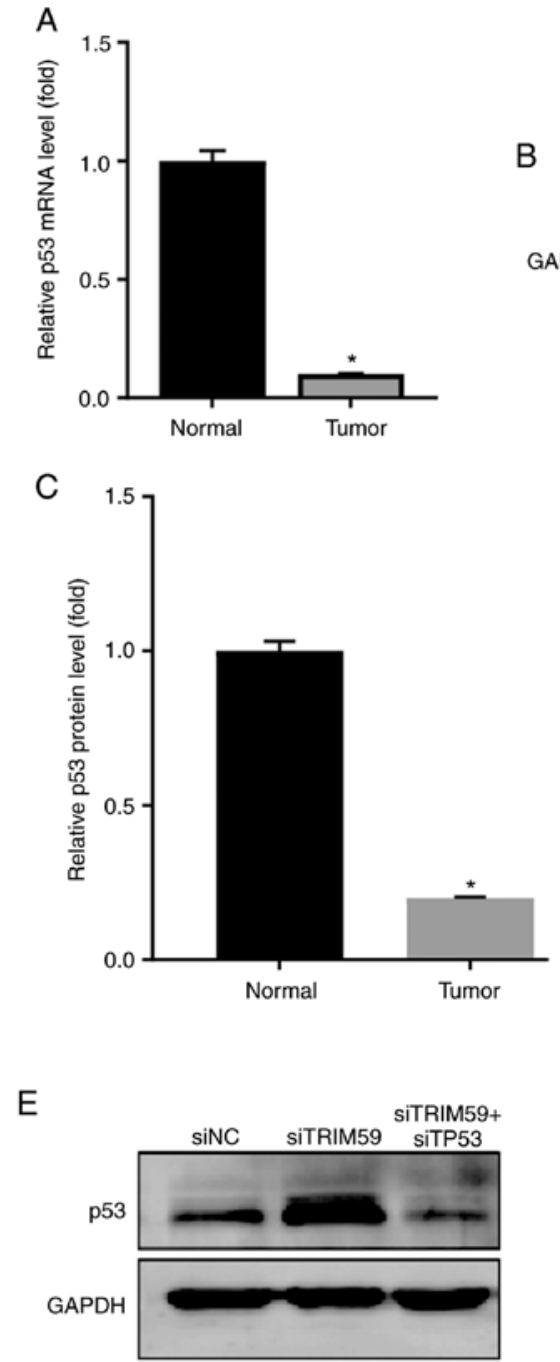

$$
\text { G }
$$
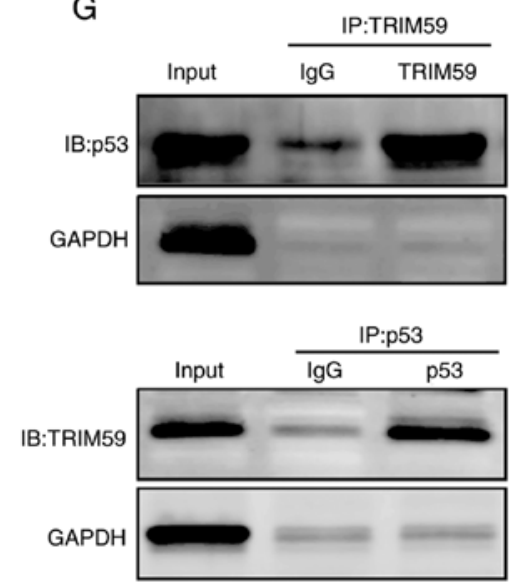
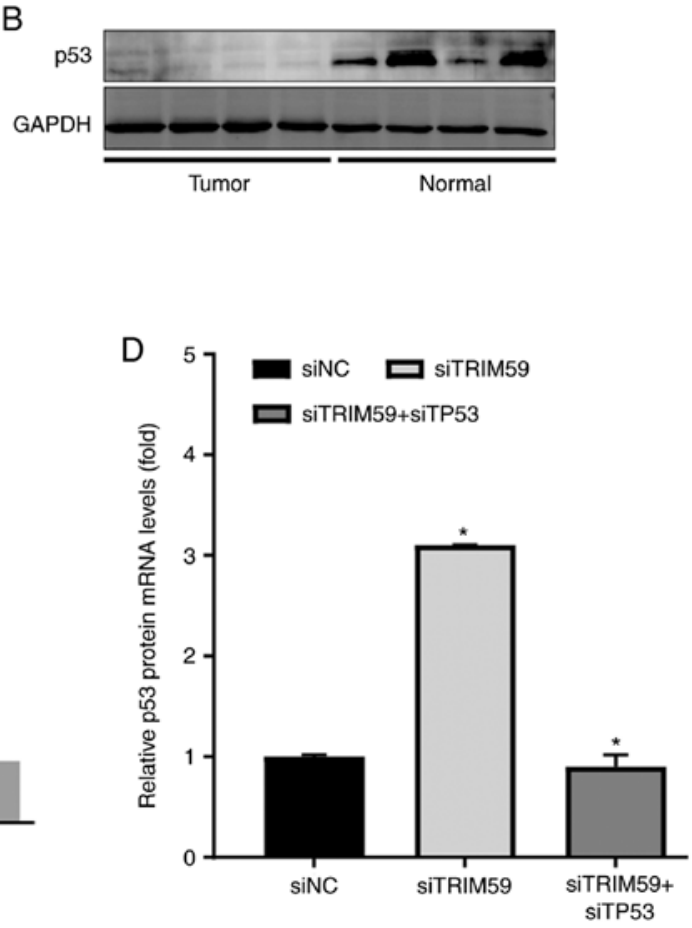

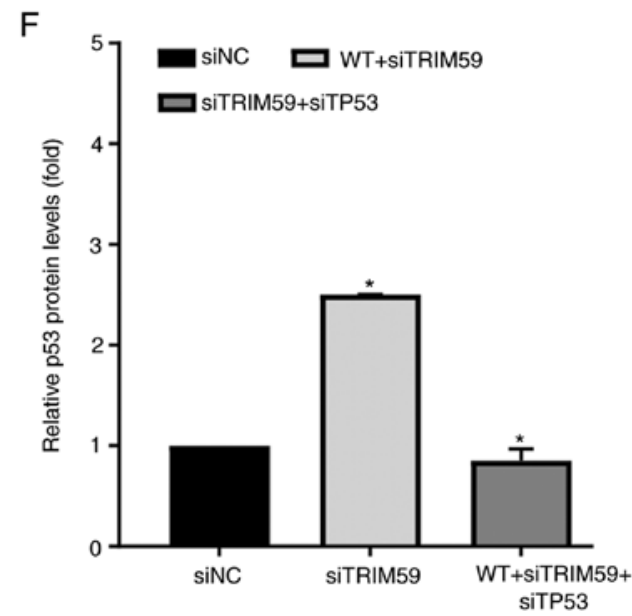

Figure 5. TRIM59 expression is negatively associated with p53 in tumor tissues and Eca109 cells. (A) p53 mRNA expression levels in tumor and matched healthy tissues. p53 protein expression levels were (B) determined via western blotting and (C) semi-quantified. (D) At $48 \mathrm{~h}$ post-transfection, p53 mRNA expression levels were detected in siTRIM59- and siTP53-transfected Eca109 cells. (E) At 48 h post-transfection, p53 protein expression levels were (E) determined via western blotting and (F) semi-quantified in siTRIM59- and siTP53-transfected Eca109 cells. (G) TRIM59 co-immunoprecipitated with p53 in 293T cells transfected with 553 and TRIM59. "P<0.05. vs. healthy or siNC. TRIM59, tripartite motif 59; si, small interfering RNA; TP53, tumor protein p53; NC, negative control; WT (reference to cells with no treatment), wild-type.

types of cancer, such as lung (17,18) and gastric cancer (19). TRIM59 protein functions as an E3 ubiquitin ligase, and is comprised of an N-terminal really interesting RING-finger domain (1). A previous study analyzed TRIM59 expression profiles in different types of cancer, and demonstrated that TRIM59 was markedly upregulated across 12 cancer types (12). A correlation between higher expression levels of TRIM59 in cancer tissues, and particularly its involvement in advanced stages of malignant transformation, and poor patient prognosis was identified. Previous studies have reported that 
TRIM59-silencing could decrease the growth rate of lung cancer, prostate cancer $(20)$, breast cancer $(21,22)$, colorectal cancer (23), cholangiocarcinoma (24), human cervical cancer (25), gliomagenesis (26,27), medulloblastoma (28), ovarian cancer $(29,30)$ and gastric cancer $(19)$. The aforementioned studies indicated that TRIM59 may function as an oncogene in the majority of cancer types (31).

However, whether the TRIM59 protein promotes hESC proliferation and migration, and whether the increased protein expression of TRIM59 is correlated with poor prognosis in patients with hESC is not completely understood. In the present study, TRIM59 was upregulated in hESC tissues and cell lines compared with adjacent healthy tissues and cell lines. Following TRIM59 knockdown in Eca109 cells, cells displayed significantly lower viability and migration, as well as reduced colony formation ability compared with the siNC group. Moreover, higher protein expression of TRIM59 in patients with hESC was associated with a significantly poorer prognosis, as determined by Kaplan-Meier analysis. The results indicated that TRIM59 was associated with hESC tumorigenesis and migration, and may function as an oncogene.

Cisplatin is the most common anticancer drug used to inhibit cancer proliferation and migration (11). However, due to the long time period required for clinical chemotherapeutic treatment with cisplatin, cancer cells develop chemoresistance to the drug, and its efficacy diminishes, resulting in cancer treatment failure $(32,33)$. In the present study, siTRIM59 was used to knock down the protein expression of TRIM59 in Eca109 esophageal cancer cells, which were then exposed to cisplatin. Treatment with siTRIM59 and cisplatin decreased cell viability and increased apoptosis to a greater level compared with cells treated with siTRIM59 or cisplatin alone. To study the effectiveness of the combined treatment of siTRIM59 and cisplatin, the protein expression levels of MMP2 and MMP9 was detected. The results suggested that MMP2 and MMP9 expression levels were downregulated in the si-TRIM59 + cisplatin group compared with the siTRIM59 group. To the best of our knowledge, the present study was the first to report the effectiveness of the combination of siTRIM59 and cisplatin in hESC.

In the present study, TP53 protein expression was decreased in hESC tissues compared with adjacent healthy tissues. TP53 is one of the most important tumor suppressors and the first line of defense that keeps the genome of several types of cells stable by preventing genome changes (6). In humans, activation of p53 is a vital regulator of post-target resistance to chemotherapy and can induce cell apoptosis in the presence of strong survival signals $(6,14)$. Cells with decreased p53 protein expression are unable to respond in a timely manner to cellular stress and are more prone to a decreased response to chemotherapeutic drugs and radiotherapy, resulting in cancer migration (6). In the present study, TRIM59 knockdown increased p53 protein expression and enhanced the effective anticancer functions in Eca109 cells compared with the siNC group. Co-immunoprecipitation was used to detect the direct action by transfecting $293 \mathrm{~T}$ cells with TRIM59 and p53. The results suggested that TRIM59 promoted p53 degradation, resulting in hESC cell proliferation and metastasis via direct bonding. Therefore, the results indicated that the combination of TRIM59 inhibitor and cisplatin might serve as a novel therapeutic strategy for hESC.

In conclusion, the present study suggested that upregulation of TRIM59 in hESC tissues was associated with poor patient prognosis, and TRIM59 may serve as an oncogene to promote the proliferation and metastasis of hESC. The present study also indicated that TRIM59 knockdown inhibited Eca109 cancer cell proliferation and migration, and enhanced chemosensitivity to cisplatin by increasing p53 expression. Collectively, the results indicated that the combination of TRIM59 knockdown and cisplatin treatment may serve as a promising therapeutic strategy for patients with hESC.

\section{Acknowledgements}

Not applicable.

\section{Funding}

The present study was supported by a grant from the Key Research Program of Hebei Science and Technology committee in China (grant no. 182777206).

\section{Availability of data and materials}

The datasets used and/or analyzed during the present study are available from the corresponding author upon reasonable request.

\section{Authors' contributions}

JL and ZW conceived and designed the study. RL performed the experiments and wrote the manuscript. HL performed statistical analysis and revised the manuscript for intellectual content. YX and XG followed up the patients and performed the immunofluorescence experiments. YC and XL performed bioinformatics analysis. JS contributed to the design and analysis of immunoprecipitation experiments. All authors read and approved the final manuscript.

\section{Ethics approval and consent to participate}

The present study was approved by the Ethics Committee of the Forth Hospital of Hebei Medical University (Hebei, China; approval no. Science Research-2014-Oncology-99). Written informed consent was provided by all patients prior to the study start.

\section{Patient consent for publication}

Not applicable.

\section{Competing interests}

The authors declare that they have no competing interests.

\section{References}

1. Esposito D, Koliopoulos MG and Rittinger K: Structural determinants of TRIM protein function. Biochem Soc Trans 45: 183-191, 2017.

2. Hatakeyama S: TRIM family proteins: Roles in autophagy, immunity, and carcinogenesis. Trends Biochem Sci 42: 297-311, 2017. 
3. Hatakeyama S: TRIM proteins and cancer. Nat Rev Cancer 11: 792-804, 2011.

4. Nabeshima K, Inoue T, Shimao Y and Sameshima T: Matrix metalloproteinases in tumor invasion: Role for cell migration. Pathol Int 52: 255-264, 2002.

5. Scheau C, Badarau IA, Costache R, Caruntu C, Mihai GL, Didilescu AC, Constantin C and Neagu M: The role of matrix metalloproteinases in the epithelial-mesenchymal transition of hepatocellular carcinoma. Anal Cell Pathol (Amst) 94: 23907, 2019.

6. Yanqing L, Omid T and Wei G: p53 modifications: Exquisite decorations of the powerful guardian. J Mol Cell Biol 11: 564-577, 2019.

7. Malkin D: Li-fraumeni syndrome. Genes Cancer 2: 475-484, 2011.

8. Guha T and Malkin D: Inherited TP53 mutations and the Li-Fraumeni syndrome. Cold Spring Harb Perspect Med 7: a026187, 2017.

9. Freddie B, Jacques F, Isabelle S, Rebecca SL, Lindey TA and Ahmedin J: Global cancer statistics 2018: GLOBOCAN estimates of incidence and mortality worldwide for 36 cancers in 185 countries. CA Cancer J Clin 68: 394-424, 2018.

10. Jackie Oh S, Han S, Lee W and Lockhart AC: Emerging immunotherapy for the treatment of esophageal cancer. Expert Opin Investig Drugs 25: 667-677, 2016.

11. Zhang F, Wang Y, Wang ZQ, Sun P, Wang DS, Jiang YX, Zhang DS, Wang FH, Xu RH and Li YH: Efficacy and safety of cisplatin-based versus nedaplatin-based regimens for the treatment of metastatic/recurrent and advanced esophageal squamous cell carcinoma: A systematic review and meta-analysis. Dis Esophagus 30: 1-8, 2017.

12. Tan P, Ye Y, He L, Xie J, Jing J, Ma G, Pan H, Han L, Han W and Zhou Y: TRIM59 promotes breast cancer motility by suppressing p62-selective autophagic degradation of PDCD10. PLoS Biol 16: e3000051, 2018

13. Wang Z, Li C, Li Y, Guo X, Yan Z, Gao F and Li C: DpdtbA-induced growth inhibition in human esophageal cancer cells involved inactivation of the p53/EGFR/AKT pathway. Oxid Med Cell Longev 2019: 5414670, 2019.

14. Valletti A, Marzano F, Pesole G, Sbisa E and Tullo A: Targeting chemoresistant tumors: Could trim proteins-p53 axis be a possible answer? Int J Mol Sci 20: 1776, 2019.

15. Siegel RL, Miller KD and Jemal A: Cancer Statistics, 2017. CA Cancer J Clin 67: 7-30, 2017

16. Rustgi A and El-Serag HB: Esophageal carcinoma. N Engl J Med 372: 1472-1473, 2015.

17. Hao L, Du B and Xi X: TRIM59 is a novel potential prognostic biomarker in patients with non-small cell lung cancer: A research based on bioinformatics analysis. Oncol Lett 14: 2153-2164, 2017.

18. Cui Z, Liu Z, Zeng J, Zhang S, Chen L, Zhang G, Xu W, Song L and Guo X: TRIM59 promotes gefitinib resistance in EGFR mutant lung adenocarcinoma cells. Life Sci 224: 23-32, 2019.

19. Luo D, Wang Y, Huan X, Huang C, Yang C, Fan H, Xu Z and Yang L: Identification of a synonymous variant in TRIM59 gene for gastric cancer risk in a Chinese population. Oncotarget 8: $11507-11516,2017$
20. Lin WY, Wang H, Song X, Zhang SX, Zhou PS, Sun JM and Li JS: Knockdown of tripartite motif 59 (TRIM59) inhibits tumor growth in prostate cancer. Eur Rev Med Pharmacol Sci 20: 4864-4873, 2016

21. Zhang Y and Yang W: Down-regulation of tripartite motif protein 59 inhibits proliferation, migration and invasion in breast cancer cells. Biomed Pharmacother 89: 462-467, 2017.

22. Tan P, He L and Zhou Y: TRIM59 deficiency curtails breast cancer metastasis through SQSTM1-selective autophagic degradation of PDCD10. Autophagy 15: 747-749, 2019.

23. Wu W, Chen J, Wu J, Lin J, Yang S and Yu H: Knockdown of tripartite motif-59 inhibits the malignant processes in human colorectal cancer cells. Oncol Rep 38: 2480-2488, 2017.

24. Shen H, Zhang J, Zhang Y, Feng Q, Wang H, Li G, Jiang W and Li X: Knockdown of tripartite motif 59 (TRIM59) inhibits proliferation in cholangiocarcinoma via the PI3K/AKT/mTOR signalling pathway. Gene 698: 50-60, 2019.

25. Aierken G, Seyiti A, Alifu M and Kuerban G: Knockdown of tripartite-59 (trim59) inhibits cellular proliferation and migration in human cervical cancer cells. Oncol Res 25: 381-388, 2017.

26. Chen G, Chen W, Ye M, Tan W and Jia B: TRIM59 knockdown inhibits cell proliferation by down-regulating the Wnt/ $\beta$-catenin signaling pathway in neuroblastoma. Biosci Rep 39: BSR20181277, 2019

27. $\mathrm{Xu} \mathrm{Y,} \mathrm{Zhang} \mathrm{Z}$ and $\mathrm{Xu}$ G: TRIM proteins in neuroblastoma. Biosci Rep 39: BSR20192050, 2019.

28. Gao R, Lv G, Zhang C, Wang X and Chen L: TRIM59 induces epithelial-to-mesenchymal transition and promotes migration and invasion by PI3K/AKT signaling pathway in medulloblastoma. Oncol Lett 15: 8253-8260, 2018

29. Wang Y, Zhou Z, Wang X, Zhang X, Chen Y, Bai J and Di W: Trim59 is a novel marker of poor prognosis and promotes malignant progression of ovarian cancer by inducing annexin a2 expression. Int J Biol Sci 14: 2073-2082, 2018.

30. Zhang P, Zhang H, Wang Y, Zhang P and Qi Y: Tripartite motif-containing protein 59 (TRIM59) promotes epithelial ovarian cancer progression via the focal adhesion kinase(FAK)/AKT/Matrix metalloproteinase (MMP) pathway. Med Sci Monit 25: 3366-3373, 2019.

31. Khatamianfar V, Valiyeva F, Rennie PS, Lu W, Yang BB, Bauman G, Moussa M and Xuan JW: TRIM59, a novel multiple cancer biomarker for immunohistochemical detection of tumorigenesis. BMJ Open 2: e001410, 2012.

32. Huang XP, Li X, Situ MY, Huang LY, Wang J, He T, Yan QH, Xie XY,Zhang YJ, Gao YH, et al: Entinostat reverses cisplatin resistance in esophageal squamous cell carcinoma via down-regulation of multidrug resistance gene 1. Cancer Lett 414: 294-300, 2018.

33. Ilson DH: Esophageal cancer chemotherapy: Recent advances. Gastrointest Cancer Res 2: 85-92, 2008.

This work is licensed under a Creative Commons Attribution-NonCommercial-NoDerivatives 4.0 International (CC BY-NC-ND 4.0) License. 